\title{
Friction Regimes in the Lubricants Solid-State Regime
}

\author{
D. J. Schipper and O. Maathuis
}

University of Twente, Dept. Mechanical Engineering, Tribology section, Postbox 217, 7500 AE Enschede

\begin{abstract}
.
Friction measurements were performed in the lubricant's solid-state regime to study the transition from full-film lubrication, in which the separation is maintained by a solidified lubricant, to mixed lubrication. Special attention is paid to the influence of temperature (inlet viscosity) and roughness on this transition.

The friction measurements showed that in the lubricants solid-state region three lubrication modes can be distinguished: A) full-film lubrication; separation is maintained by a solid film (S-EHL), B) mixed lubrication (ML); the load is carried by the interacting asperities as well as the lubricant (acting like a solid or as a liquid) and C) boundary lubrication (BL).

Further, the dependency of the transition S-EHL to ML on temperature (inlet viscosity) depends on the lubricant used. The transition S-EHL to ML depends slightly more on roughness as found for L-EHL to ML transition.

Finally, the film thickness formulas developed for EHL contacts in which the lubricant behaves as a liquid become doubtful when operating in the lubricant solid-state regime. This on the basis of the calculated film thickness over roughness ratios for the transition S-EHL to ML.
\end{abstract}

\section{Introduction.}

From 11 terature it is known that lubricants behave as a function of temperature and pressure elther as a liquid or as a solid, [1]. The classical EHL-theory, developed for the situation that the lubricant operates in the lubricants liquidstate regime, resulted into diagrams by which it is possible to calculate the film thickness in lubricated concentrated contacts as a function of the operational conditions, see for instance [2], [3] and [4].

Due to developments in design it is known that components of drives, etc. become smaller and that they have to carry the same or even higher loads. As a result the contact pressures between these components are higher and therefore the rheological behaviour of the lubricants become more important, i.e. the lubricant's solid-state behaviour. Also is known that for the transition from liquid-state behaviour to solid-state behaviour the value of $\alpha \cdot \vec{p}$ is nearly constant, see [5] and [6]. For mineral oils, for instance, the value for $\alpha \cdot \bar{p}$ is equal to approx. $18.5 \pm 1$, whilst for 5P4E this value is 10.0 \pm 0.5 . When this result, i.e. a constant value of $\alpha \cdot \bar{p}$, is plotted into the regime chart of Johnson [2] or of Moes [4], for mineral oils one obtains the result as presented in Figs. 1 and 2 . In these Figs. the transition from liquid- to solid-state behaviour is represented by a dotted line. These Figs. show that a large part of the diagrams is covered by the solidstate behaviour of the lubricant. 


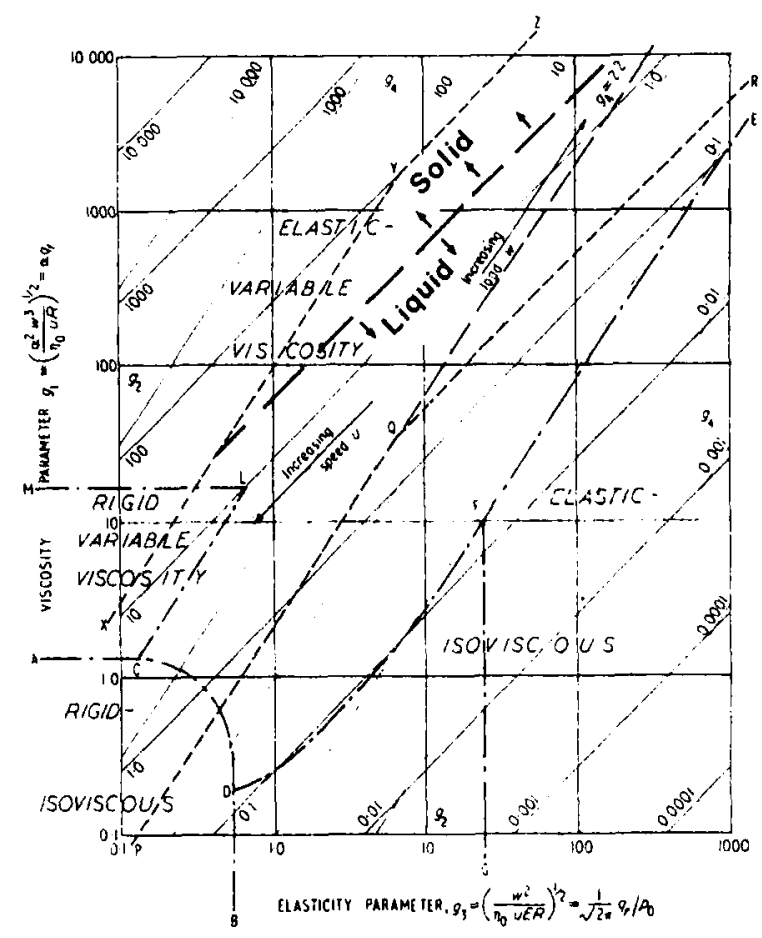

Figure 1. Regime chart of EHL [2].

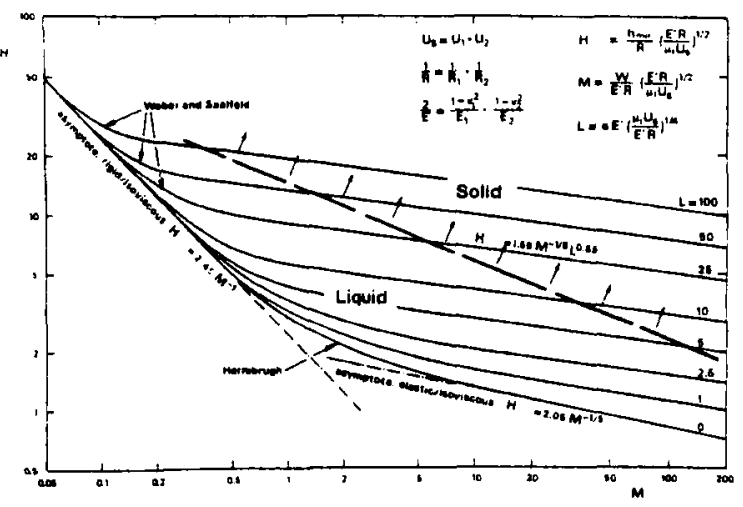

Figure 2. EHL diagram by [4].

From early research [6] on the transition between solid- and 1iquid-state behaviour of lubricants in concentrated contacts transitions were observed between fullfilm lubrication (S-EHL) and mixed lubrication (ML). It was found that this transition, i.e. S-EHL to ML occurred at very low values of $\lambda$ (ratio of film thickness over roughness), see Fig. 3.

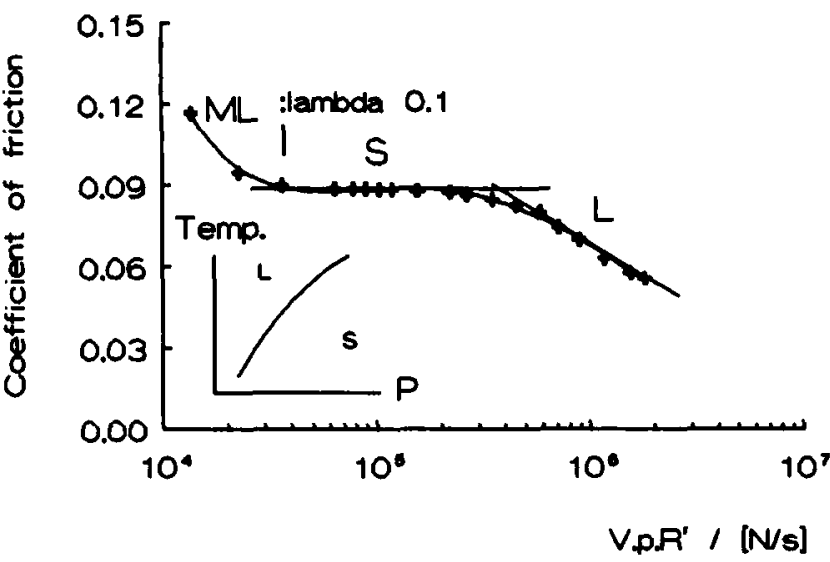

Figure 3. Friction curve obtained in the lubricants solid-state regime. HVI-650: $\bar{p}=1.5 \mathrm{GPa}, \theta=80 \mathrm{C}$.

In this figure three friction regions are distingulshed; region $L$ (L-EHL) where due to heat development the contact operates in the lubricant's liquid-state regime, region $S$ (S-EHL) where the friction is controlled by the limiting shear stress of the lubricant (solidstate behaviour) and region ML, mixed lubrication, friction is controlled by the interacting asperities and the lubricant. In region $L$ and $S$ no electric conductivity was measured, whilst in region ML electric conductivity was measured, i.e. asperity interaction.

In the present study the transition from region S-EHL to region $M L$ is invest i gated.

\section{Experimental procedure.}

\subsection{S-tribometer.}

Friction experiments were performed on a tribometer, see Fig. 4, in which a pin slides in a reciprocative way against a stationary plate.

The plate is held in a holder, mounted on a thermo-controlled heat exchanger, which in turn is supported by two steel blade springs 


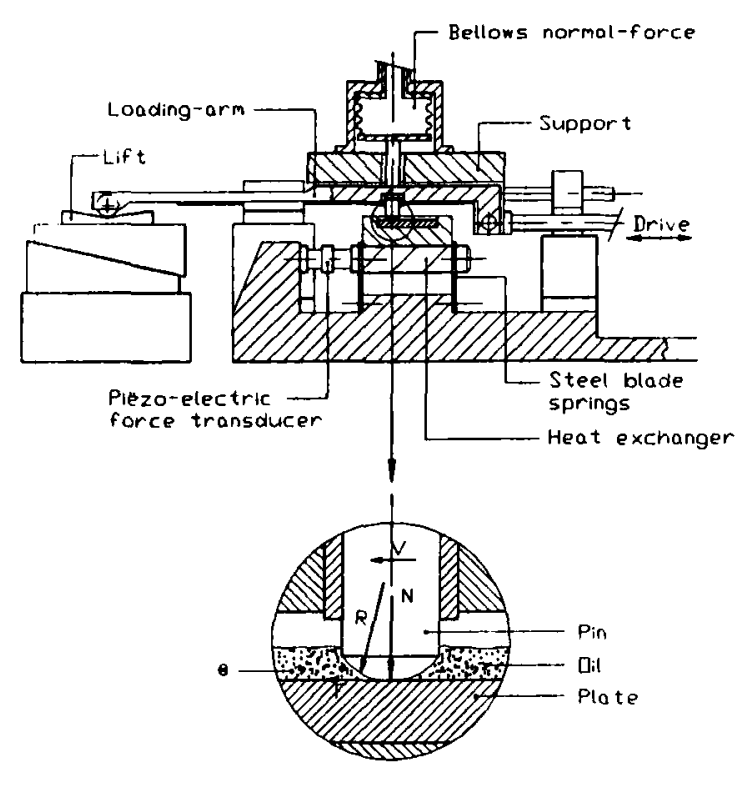

Figure 4. S-Tribometer.

which only allow movement in sliding direction. The frictional force is measured by a piezoelectric force transducer.

The pin is fixed in a loading arm, which is connected to the support. The pivot of this loading arm with support lies in the plane of the sliding contact. The support, guided by two cylindrical bars, supported by a system of 4 rollblocks, is driven by a crank mechanism, by which the support and thereby the pin exerts a sinusoidal motion with an amplitude $A=23 \mathrm{~mm}$. The $\max$. velocity is; $V=2 \pi \cdot A \cdot f$, in which $f$ is the frequency of the moving support. At the end of the loading arm a mechanical lift has been mounted on the frame. This lift makes it possible to control the length of contact between pin and plate during one cycle. As a result, the sliding velocity during contact is almost constant.

By pressurizing the bellows on top of the support, the pin is loaded against the plate. The pressure is kept constant in the bellows by using a big air-accumulator, which is connected to the bellows. The bellows pressure is measured by an accurate manometer or a piezoelectric resistive transducer. With the signal from an electro-magnetic pickup both the frequency of the moving support is measured by means of a frequency meter and the number of cycles by an electro mechanical counter. The temperature of the lubricant and plate is measured with Ni-CrNi thermocouples.

The frictional force, measured by the piezo-electric force transducer, is recorded using a digital oscilloscope. In Fig. 5 the friction force and the velocity during one cycle are schematically shown.

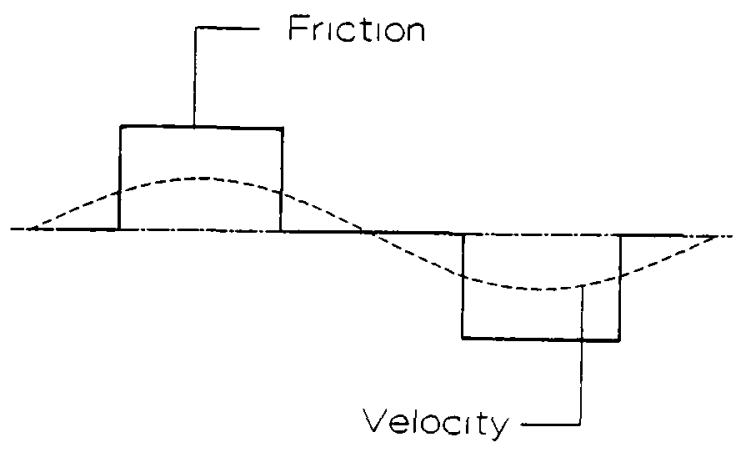

Figure 5. Friction and velocity during one cycle.

Fig. 5 shows that friction is zero during a part of the cycle and rises steeply when the roll does not make contact with the lift; contact between pin and plate is made. The frictional force decreases very fast when the roll (connected with the loading arm) is 1 ifted.

\subsection{Specimens.}

All specimens used were made of hardened (HRc $\approx 63$ ) steel AISI- 
52100. The pins were balls from roller bearings. The plates were made from AISI-52100, hardened and subsequently grinded and polished. Before each experiment the specimens were rinsed in an ultrasonic cleaner with ethane and subsequently dried in hot air. The roughness of the specimens were measured (cut-off length $0.8 \mathrm{~mm}$ ) by using an interference microscope, Promap 512.

\subsection{Lubricants.}

The experiments were performed using a mineral oil HVI -650 and a polyphenol ether 5P4E as lubricants. In the appendix some properties of these fluids are given.

\subsection{Procedure.}

A friction experiment is conducted under constant operational conditions of pressure and temperature while changing the velocity. By the right combination of pressure and temperature the friction experiment is performed in the lubricants solid-state regime. Each measured friction value is obtained after a sliding time of at least 5 minutes.

\section{Experimental results.}

3.1. Friction curves.

The result of a friction experiment performed with HVI-650 is given in Fig. 6, where the coefficient of friction is presented as a function of the velocity. Fig. 6 shows the different lubrication regions, i.e. Region L - L-EHL, Region $S$ the afore mentioned S-EHL, Region ML - mixed lubrication, and Region BL - boundary lubrication. When performing an experiment with 5P4E one obtains Fig. 7. In this figure a transition can be recognized from region $S$, where the coefficient of friction is equal to

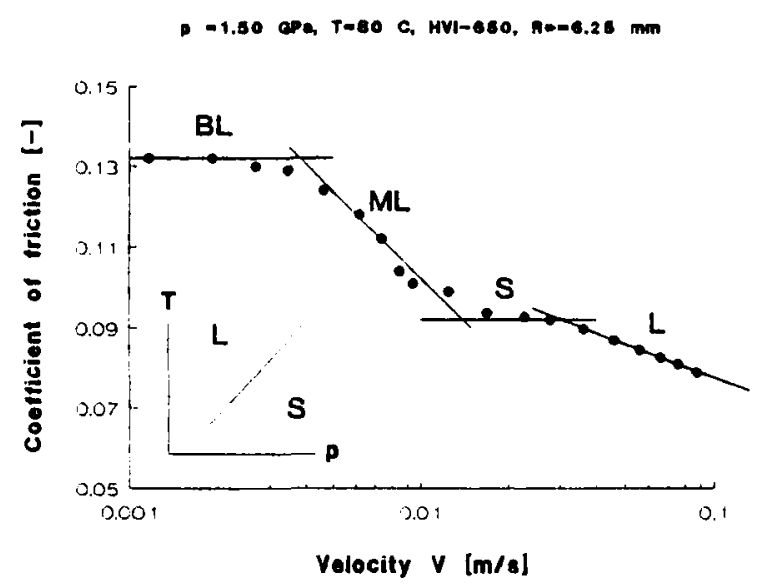

Figure 6. Friction as a function of velocity operating in the solidstate regime of HVI-650.

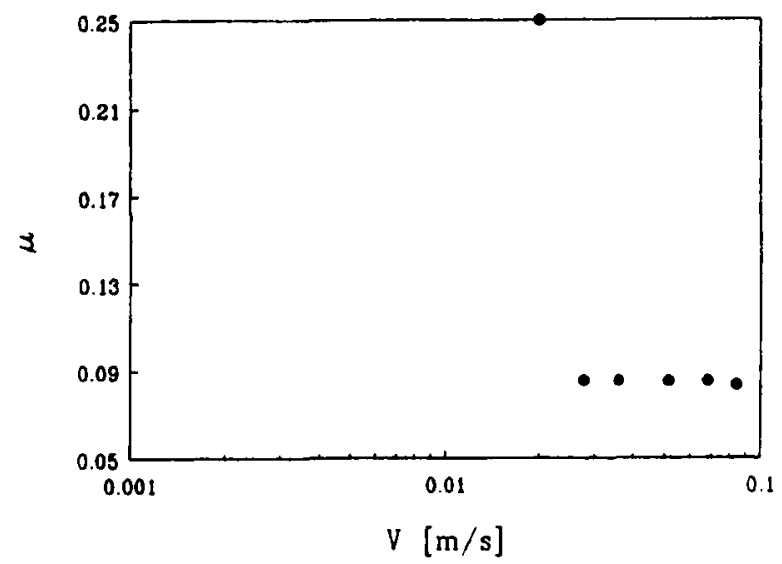

Figure 7. Friction as a function of velocity operating in the solidstate regime of $5 \mathrm{P} 4 \mathrm{E}$.

$\tau_{1} / \bar{p}$, to values characteristic for locally unlubricated contact. In this investigation $5 \mathrm{P} 4 \mathrm{E}$ is used to discriminate the transition fullfilm to mixed lubrication very well due to the fact that 5P4E does not form good boundary layers. Thus, when asperity interaction takes place, friction immediately rises to high values. In these situations a high electrical conductivity is measured, indicating metallic contact between the opposing surfaces. 


\subsection{Influences of temperature and roughness.}

The temperature was changed, while operating in the lubricants solid-state regime, to study the effect of the lubricant viscosity, in the inlet, on the transition S-EHL and ML. The temperature is expected not to have much influence on the properties in the contact, the dependency of the limiting shear stress on temperature is small. In Fig. 8 the results are given of HVI-650 and $5 \mathrm{P} 4 \mathrm{E}$.
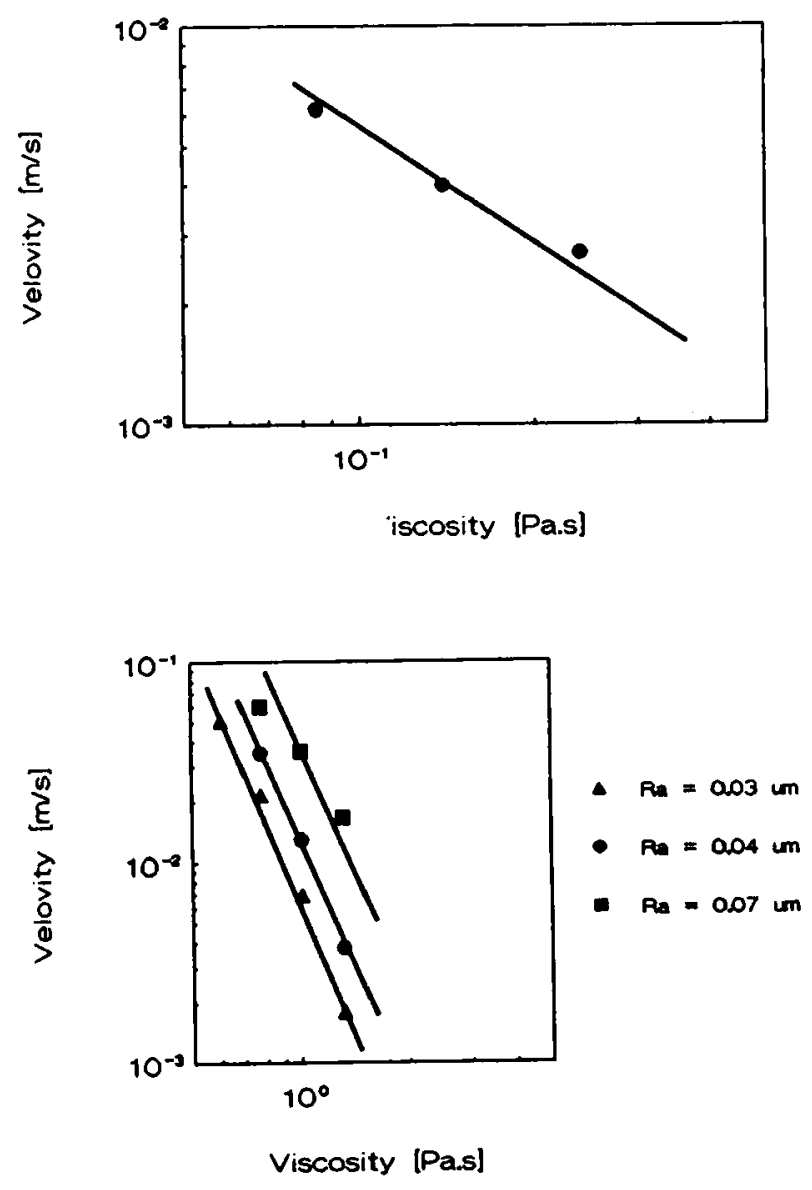

Figure 8. Transition velocity S-EHL to $M$ as a function viscosity for a) HVI-650 and b) 5P4E.
In Fig. 8 the transition velocity S-EHL to ML is plotted as a function of (inlet) viscosity. From this figure it follows that the transition lines can be expressed by $v \cdot \eta^{n}=$ const., the value of $n$ is equal to -1 and -4 for HVI -650 and 5P4E respectively. This means that the transition S-EHL to ML using 5P4E is much more sensitive with respect to temperature (viscosity) then HVI-650. This might be caused by solidification aspects of $5 \mathrm{P} 4 \mathrm{E}$ in the inlet. The power of -1 for HVI-650 is in line with results obtained from experiments performed in the lubricants liquid-state regime [5].

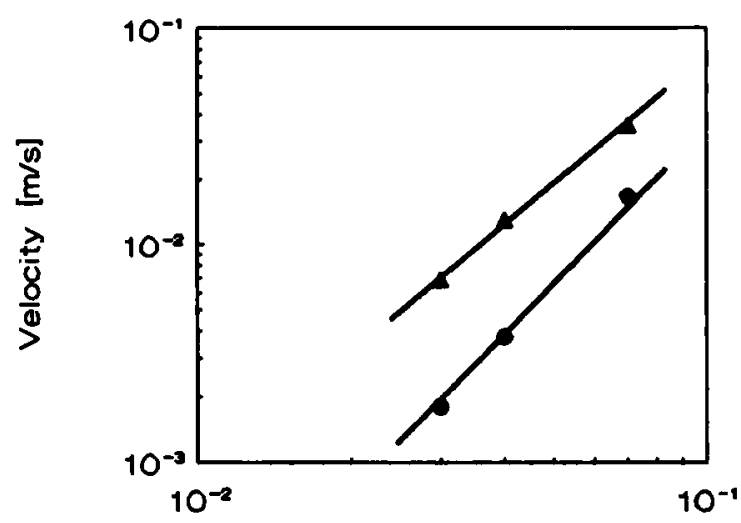

Roughness [um]

Figure 9. Transition velocity as a function of roughness.

In Fig. 9 the effect of roughness on the transition velocity S-EHL to $M L$ is given. These results can be expressed by $V \cdot R_{a}{ }^{n}=$ const.. For both lubricants a value for $n$ of approximately 2 is found. This result shows that the transition S-EHL to ML is slightly more dependent on roughness than the transition L-EHL to ML.

\section{Discussion.}

The results show that the transition S-EHL to ML with 5P4E 
strongly depends on (inlet) temperature. From the work of Gecim and Winer [7] it is known that 5P4E may solidify already in the inlet. Decreasing temperature increases the solidifled area in the inlet. As a result a thicker film or separation may exists between the two opposing surfaces [7]. Therefore, a much lower velocity is needed before contact between the two opposing surfaces occurs.

The results obtained with respect to the influence of temperature on the transition S-EHL to ML with HVI-650 is in line with results obtalned under liquid-state behaviour conditions, 1 .e. $V \cdot \eta=$ const. . However, the calculated lambda ratio's for the transition S-EHL to ML give very low values, down to 0.08 . In table 1 the range of calculated film thickness [8] over roughness values are given.

Table 1: $\lambda$ ratios for the transition S-EHL and ML.

\begin{tabular}{|ccc|}
\hline & HVI -650 & 5P4E \\
\hline$\lambda$ & $0.08-0.42$ & $0.22-4.0$ \\
\hline
\end{tabular}

Normally, 1.e, such operational conditions that the lubricant behaves like a liquid, $\lambda$ values for the transition L-EHL to ML of 1 are expected, [9]. For the very first asperity interactions a value of $\lambda$ of 3 is assumed [9]. From table 1 it is clear that under solid-state behaviour of the lubricant this definition does not hold. On the basis of these results it is concluded that the well known film thickness formulas developed for the L-EHL become doubtful for the
S-EHL. To the authors opinion it is necessary to analyze the rheological models for lubricants operating under conditions of solidstate behaviour in order to obtain the right predictions of film thickness under these conditions.

The dependency of the transition $S-E H L$ to $M L$ on roughness is slightly higher than for the transition L-EHL to ML. For the transition L-EHL to ML a value for $\mathrm{n}$ of 1.5 is found [5]. The results of the experiments described in this article give a value for $n$ of approx. 2 for the transition S-EHL to ML. The roughness varied in these experiments from 0.03 to 0.07 $\mu \mathrm{m}$, which is rather small.

\section{Conclusions.}

On the basis of the experiments performed the following conclusions can be drawn:

- In the lubricants solid-state region three lubrication modes can be distinguished:

- Full-film lubrication; separation by a solid film (S-EHL).

- Mixed lubrication (ML); the lubricant behaves like a liquid or as a solid.

- Boundary lubrication (BL); the lubricant behaves like a liquid.

- The dependency of the transition S-EHL to ML on temperature (inlet viscosity) depends on the lubricant used.

- The transition S-EHL to ML depends slightly more on roughness as found for the L-EHL to ML transition.

- The film thickness formulas developed for EHL contacts in which the lubricant behaves as a liquid become doubtful when operating in the lubricant solid-state regime. 
Acknowledgements.

The authors are grateful to $\mathrm{Mr}$. E.G. de Vries for keeping the tribotester operational.

\section{References.}

1. Alsaad, M., Bair, S., Sanborn D. and Winer, W.O., ASME J. of Lubr. Techn., Vol. 100 (1978), pp. 404-417.

2. Johnson, K.L., J. Mech. Eng. Sci., Vol. 12 (1970), pp 9-16.

3. Greenwood, J.A. , Proc. Instn. Mech. Engrs., Vol. 202 (1988), no. C1, pp 11-17.

4. Moes, H. , Proc. Instn. Mech. Engrs., Vol. 180 (1965-66), Part 3B, pp 244-245.

5. Schipper, D. J., Ph. D. Thesis, Univ. of Twente, 1988, Enschede, The Netherlands.

6. Schipper, D. J., Vroegop, P.H. and Gee, A.W.J., Proc. of the Japan International Tribology Conference, Nagoya, Japan, 1990, Vol. 1, pp. 379-384.

7. Gecim, B. and Winer, W.O., ASME $\mathrm{J}$. of Lubr. Techn., Vol. 102 (1980), p 213.

8. Hamrock, B.J. and Dowson, D., ASME J. of Lubr. Techn., Vol. 99 (1977), pp. 264-276.

9. Bair, S. and Winer, W. O., ASME $\mathrm{J}$. of Lubr. Techn., Vol. 104 (1982), pp. 382-391.

10 Evans, C.R., Ph. D. Thesis, Univ. of Cambridge, 1983, UK.

\section{Nomenclature.}

$\bar{p} \quad$ Mean Hertzian contact pressure.

R* Reduced radius

Ra Combined CLA surface roughness,

$R a=\left(R a_{1}{ }^{2}+R a_{2}{ }^{2}\right)^{1 / 2}$

V Velocity.

$[\mathrm{m}]$

$\begin{array}{ll}\eta & \text { Viscosity. } \\ \theta & \text { Temperature. }\end{array}$

[Pa.s]

$\left[{ }^{\circ} \mathrm{C}\right]$ $\bar{\tau}_{l} \quad$ Limiting shear stress [Pa]

$\mu$ Coefficient of friction [-]

Appendix: Lubricant properties.

Viscosity, $\eta$, in $\mathrm{mPa} \cdot \mathrm{s}$

\begin{tabular}{|c|cc|}
\hline $\begin{array}{c}\text { Temperat ure } \\
{ }^{\circ} \mathrm{C}\end{array}$ & $\begin{array}{c}\text { SANTOVAC-5* } \\
\text { 5P4E }\end{array}$ & HVI-650* \\
\hline 40 & 266 & 450 \\
60 & 79 & 140 \\
80 & 31 & 55 \\
100 & 15 & 27 \\
\hline
\end{tabular}

Limiting shear stress and pressure.

\begin{tabular}{|l|cc|}
\hline & $\begin{array}{c}\text { SANTOVAC-5 } \\
5 \text { HVE }\end{array}$ & HV -650 \\
\hline $\begin{array}{l}\text { Literature } \\
\bar{\tau}_{l} / \overline{\mathrm{p}} 40^{\circ} \mathrm{C}\end{array}$ & $\begin{array}{c}6.8 \cdot 10^{-2} \\
\left(40-100^{\circ} \mathrm{C}\right)\end{array}$ & $9.0 \cdot 10^{-2}$ \\
Measured & & \\
$\bar{\tau}_{1} / \overline{\mathrm{p}} 40^{\circ} \mathrm{C}$ & $8.7 \cdot 10^{-2}$ & $9.2 \cdot 10^{-2}$ \\
$\bar{\tau}_{1} / \overline{\mathrm{p}} 80$ & - & $8.6 \cdot 10^{-2}$ \\
\hline
\end{tabular}

Liquid- to solid-state transition.

\begin{tabular}{|c|c|}
\hline & $\begin{array}{l}\text { SANTOVAC-5*"HVI-650** } \\
\text { 5P4E }\end{array}$ \\
\hline$\alpha \cdot \bar{p}$ & $10.0 \pm 0.5 \quad 18.5 \pm 1$ \\
\hline
\end{tabular}

* Evans, [10]
Schipper, [5] 